\title{
Critical Concentration
}

National Cancer Institute

\section{Source}

National Cancer Institute. Critical Concentration. NCI Thesaurus. Code C73488.

The concentration of a solute or dispersion above which spontaneous aggregation or precipitation occurs. 